\title{
Chronic heart failure in Japan: Implications of the CHART studies
}

\author{
Nobuyuki Shiba \\ Hiroaki Shimokawa \\ Department of \\ Cardiovascular Medicine, \\ Department of Evidence-Based \\ Cardiovascular Medicine, Tohoku \\ University Graduate School of \\ Medicine, Sendai City, Japan
}

\begin{abstract}
The prognosis of patients with chronic heart failure (CHF) still remains poor, despite the recent advances in medical and surgical treatment. Furthermore, CHF is a major public health problem in most industrialized countries where the elderly population is rapidly increasing. Although the prevalence and mortality of CHF used to be relatively low in Japan, the disorder has been markedly increasing due to the rapid aging of the society and the Westernization of lifestyle that facilitates the development of coronary artery disease. The Chronic Heart Failure Analysis and Registry in the Tohoku District (CHART)-1 study was one of the largest cohorts in Japan. The study has clarified the characteristics and prognosis of Japanese patients with CHF, demonstrating that their prognosis was similarly poor compared with those in Western countries. However, we still need evidence for the prevention and treatment of CHF based on the large cohort studies or randomized treatment trials in the Japanese population. Since the strategy for CHF management is now changing from treatment to prevention, a larger-size prospective cohort, called the CHART-2 study, has been initiated to evaluate the risk factors of CHF in Japan. This review summarizes the current status of CHF studies in Japan and discusses their future perspectives.
\end{abstract}

Keywords: heart failure, aging, Japanese

\section{Introduction}

Chronic heart failure (CHF) is the leading cause of mortality in most developed countries (Hunt et al 2001). The prevalence and mortality rates of CHF used to be relatively lower in Japan compared with other Western countries. In Japan, approximately 1 to 2 million patients have CHF and nearly 170,000 patients die due to heart diseases each year (approximately 130 per 100,000 person-years) (Summary of Vital Statistics 2005). However, the prevalence and death rates of cardiovascular diseases and CHF have been rapidly increasing in Japan, due to the Westernization of lifestyle, including dietary habits, and the aging population (The Status of Aging 2007). The Chronic Heart Failure Analysis and Registry in the Tohoku District (CHART)-1 study was one of the largest cohort studies with Japanese CHF patients $(\mathrm{N}=1,278)$, which was designed to evaluate the characteristics and prognosis of those patients. We have also started a new cohort study, named the CHART-2 study ( $\mathrm{N}=10,000$, expected) aiming to elucidate the effective preventive measures for CHF. This review briefly summarizes the major socio-medical issues of Japanese patients with CHF, their clinical characteristics and prognosis found in our CHART-1 study, and the current status of CHF studies in Japan.

\section{Socio-medical status of Japanese patients with CHF}

\section{Rapid aging of Japanese population}

Until the 1980s, Japan had a lower percentage of elderly citizens compared with any other developed countries. However Japan is now one of the countries in which the 
population is aging rapidly. Figure 1 shows the time-course of aging and population projections between 1950 and 2055, which is assembled using the data reported by the Japanese Cabinet Office (The Status of Aging 2007). As of October 1,2006 , the total population of Japan was 127.8 million and the number of elderly aged 65 or older was 26.6 million, accounting for $21 \%$ of the total population. The elderly population is expected to continue to increase rapidly and the percentage of the elderly will reach 35.8 million (27\%) in 2055 (The Status of Aging 2007). Life expectancy in Japan at birth has also drastically increased since World War II to 78.6 years for males and 85.5 years for females in 2005 (Life Expectancies at Specified Ages 2006). The Japanese Cabinet Office expects that it will reach 83.7 years for males and 90.3 years for female in 2055. Thus, in the near future, Japanese society will encounter more difficult medical problems due to rapid aging, which other developed countries have never before experienced.

\section{Changing causes of death in Japan}

Infectious diseases such as pneumonia, tuberculosis, and gastroenteritis were the leading causes of death in Japan until the mid 1900s. The major health problems in Japanese society have drastically changed since World War II. The morbidity and mortality rates of lifestyle-related diseases such as cancer, heart disease, stroke, and diabetes mellitus have dramatically increased. Approximately $60 \%$ of the mortality is now attributed to lifestyle-related diseases (cancer, 31\%; ischemic heart disease $16 \%$; cerebrovascular disease $13 \%$; diabetes mellitus $1 \%$; and hypertensive disease $0.6 \%$ ) and the medical costs for these diseases amounts to 10.2 trillion yen ( 87.8 billion US dollars), accounting for approximately $30 \%$ of the total cost of the Japanese health insurance in 2003 (Exercise and Physical Activity Reference for Health Promotion 2006). Currently, heart disease is the second most frequent cause of death in Japan. Figure 1 shows the trend of the mortality due to heart diseases, which is constructed using the reports of death certificates in Japan (Summary of Vital Statistics 2005). There is a clear trend for the increase in death due to heart disease since 1950s (there was a temporary sharp decline in 1995 due to the tenth revision of the International Classification of Diseases regarding the description of diagnosis in death certificates).

\section{Health insurance system and future economic burden in Japan}

In Japan, all citizens are enrolled in the mandatory health insurance system based on employment and residential status. The average number of visits to a doctor per year is 16 in Japan, versus 5.8 visits in the United States (Itoh 2004). As elderly patients tend to visit doctors more frequently and to have more medication or high-cost medical care, medical

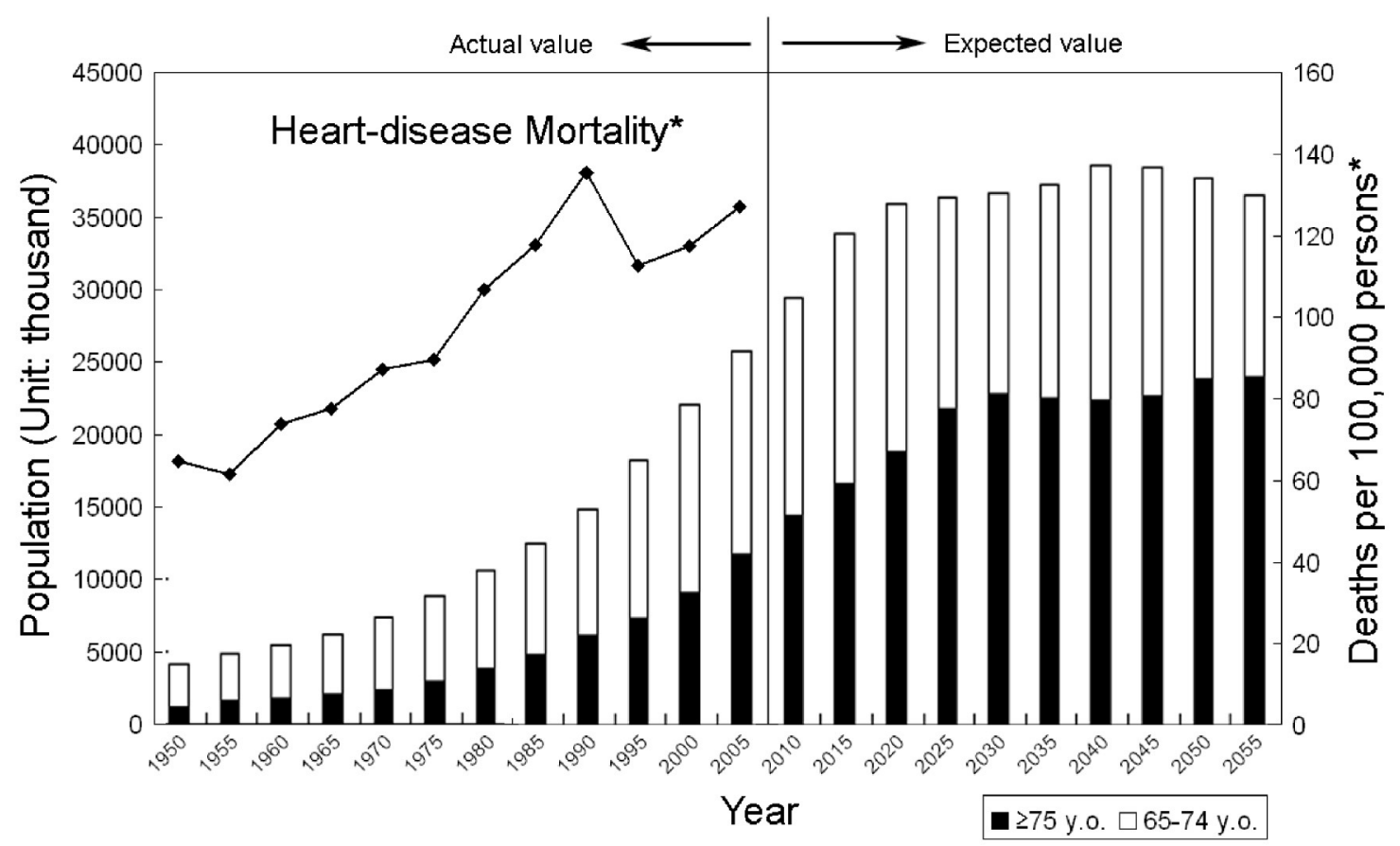

Figure I Time-course and future prediction of the increase in elderly population and heart-disease mortality in Japan. Data are based on the Status of Aging and Implementation of Measures for Aging Society in 2005, reported by the Japanese Cabinet. 
expenditure for the elderly is already taking one-third of the national health expenditure, and is projected to reach a half of the expenditure by the year 2025 (Itoh 2004). Progressive aging of the society and the consequent increase in the number of patients with $\mathrm{CHF}$ will cause more financial burden within Japanese society, which could lower the quality of medical services in the future.

\section{An overview of heart failure studies in Japan}

In Japan, medical treatment for patients with CHF is mainly based on the evidence obtained from randomized trials in the United States and Europe. There have been no sufficient randomized treatment trials or prospective cohort studies in Japan to clarify the real characteristics of Japanese patients with CHF or to improve their prognosis and quality of life. The mandatory health insurance system, the shortage of the budget to fulfill mega-trials, and the absence of trained research nurse system may all be responsible for the current situation. This section describes several cohort studies with Japanese CHF patients, major outcomes of the CHART-1 study, and randomized treatment trials for CHF performed with Japanese patients, either those that have already been published or are currently in progress. Finally, racial differences will be discussed, because this issue may also influence the impact of risk factors and/or the effects of treatments for $\mathrm{CHF}$.

\section{Prospective cohort studies in Japan}

There are few multi-institutional prospective cohort studies with CHF patients in Japan (Table 1). The Chronic Heart Failure Analysis and Registry in the Tohoku District (CHART)-1 study was the first cohort study in Japan, including more than 1,000 Japanese patients with stable CHF, who were registered at 26 hospitals in the Tohoku district with a population of approximately 9.8 million (Shiba et al 2004). The CHART-1 study was initiated in February 2000 and was completed in December 2005. The total number of CHF patients enrolled was 1,278 and the mean follow-up period was 3.5 years. Details of design and the main outcome will be presented at the following part in this article. The Japanese Cardiac Registry of Heart Failure in Cardiology (JCARE-CARD) is a registry of hospitalized patients with worsening CHF at 164 hospitals throughout the country between January 2004 and June 2005 (Tsutsui et al 2006). Death and hospital admission of the patients were followed through 2006 with the mean follow-up period of at least 1 year. Results of this study will appear in the near future. The Japanese Cardiac Registry of

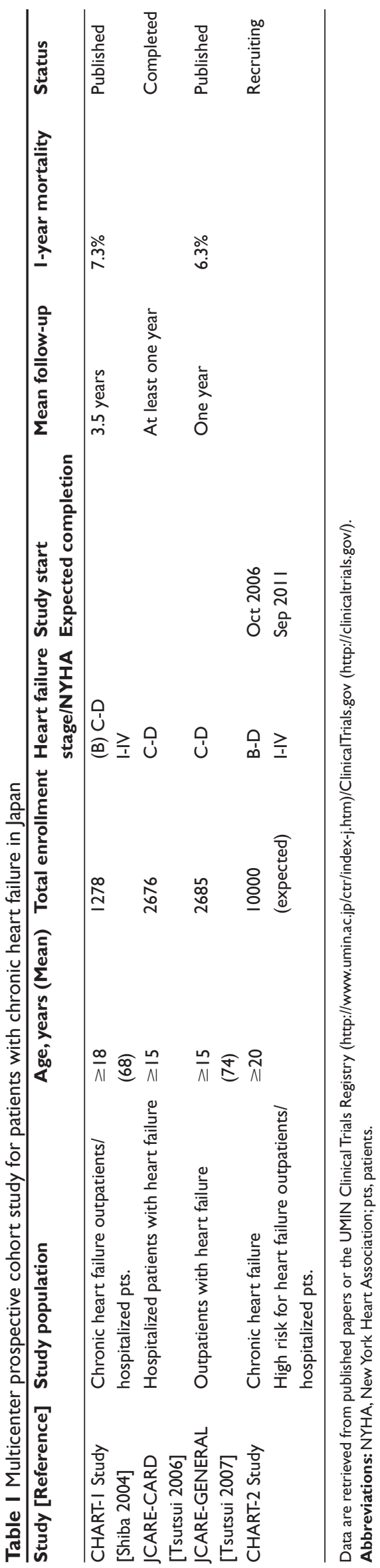


Heart Failure in General Practice (JCARE-GENERAL) is a registry of outpatients with $\mathrm{CHF}$ managed by cardiologists in hospitals and primary care physicians in general practice (Tsutsui et al 2007). Baseline data of totally 2,685 patients were collected during November 2004 and follow-up data were collected for 1 year after the enrolment. During the mean follow-up period of 427 days, the crude mortality rate was $6.7 \%$ in patients managed by cardiologists and $5.9 \%$ in those managed by general physicians. The Chronic Heart Failure Analysis and Registry in the Tohoku District (CHART)-2 study is currently the largest prospective and hospital-based cohort study with patients with CHF in Japan. This study was designed to investigate the characteristics and prognosis of a total of 10,000 patients with symptomatic CHF (Stage C/D in the ACC/AHA classification) and those with structural heart disease but without signs or symptoms of CHF (Stage-B in the AHA/ACC classification) (Hunt et al 2001). This study will elucidate the incidence and prognostic impact of metabolic syndrome in those patients, especially on the development of the first symptomatic CHF. The CHART-2 study was started in October 2006 and will be completed in September 2011.

\section{The CHART-I Study}

Risk stratification is the first line strategy to improve the prognosis and quality of life of patients with CHF. A number of factors have been found to correlate with the mortality of patients with CHF (Rector et al 1994; Deedwania 2003; Bettencourt et al 2000). The CHART-1 study was started to register patients with stable CHF in February 2000 to clarify the characteristics and prognosis and to seek for prognostic factors in Japanese CHF patients (Shiba et al 2004, 2005). Patients were enrolled when at least one of the following criteria was met: (1) left ventricular ejection fraction (LVEF) $<50 \%$, (2) left ventricular end-diastolic dimension $>55 \mathrm{~mm}$, or (3) at least one episode of congestive heart failure. Patients less than 18 years old or those with clinically unstable conditions were excluded. Baseline data, including laboratory findings, results of echocardiography, and medical treatments for $\mathrm{CHF}$, were recorded and annual surveillance was performed until the end of 2005.

\section{Characteristics and prognosis of patients with CHF in Japan.}

A total of 1,278 patients were enrolled in the CHART-1 cohort. The mean age of the study population was 68.3 years, and male accounted for $66 \%$ of the total study population. The prevalence of diabetes mellitus and hypertension was
$19 \%$ and $47 \%$, respectively. Other baseline characteristics of patients are shown in Table 2. Ischemic etiology accounted for only $25 \%$ and the percentage of patients older than 65 years was $66 \%$. Patients with preserved systolic function (defined as LVEF $>50 \%$ ) accounted for $45 \%$ of the total population. During the mean follow-up period of 3.5 years, all-cause mortality rate at 1-, 2-, and 3-year was 7\%, 16\%, and $22 \%$, respectively (Figure 2). Multivariate Cox analysis showed that several covariates, such as age, diabetes mellitus, ventricular tachycardia, serum level of B-type natriuretic peptide (BNP), rural residence, and NYHA functional class, were significantly associated with all-cause mortality (Shiba et al 2004). Figure 3 shows the Kaplan-Meier analyses of freedom from all-cause mortality in patients stratified by serum level of BNP or LVEF. Patients with higher BNP concentration had a significantly poorer prognosis (Watanabe et al 2005), however, the prognostic impact was not significantly different between patients with 200-500 pg/mL of BNP level and those with $>500 \mathrm{pg} / \mathrm{mL}$ (Figure $3 \mathrm{~A}$ ). The all-cause mortality of patients with preserved systolic function ( $\mathrm{LVEF}>50 \%$ ) was not significantly different than that of patients with

Table 2 Baseline characteristics of the Japanese patients in the CHART-I study

\begin{tabular}{|c|c|}
\hline No. of patients & $\mathrm{I}, 278$ \\
\hline Follow-up period (years) & $3.5 \pm 1.7$ \\
\hline Age (years) & $68.3 \pm 13.4$ \\
\hline$\leq 39$ & $3.7 \%$ \\
\hline $40-64$ & $30.2 \%$ \\
\hline $65-74$ & $32.8 \%$ \\
\hline$\geq 75$ & $33.3 \%$ \\
\hline Male (\%) & $66.0 \%$ \\
\hline \multicolumn{2}{|l|}{ NYHA } \\
\hline I & $19.7 \%$ \\
\hline II & $63.0 \%$ \\
\hline III & $16.5 \%$ \\
\hline IV & $0.8 \%$ \\
\hline \multicolumn{2}{|l|}{ Underlying disease } \\
\hline Coronary artery disease & $25.4 \%$ \\
\hline Valvular heart disease & $26.4 \%$ \\
\hline Left ventricular hypertrophy & $14.0 \%$ \\
\hline Non-ischemic cardiomyopathy & $28.6 \%$ \\
\hline Other & $5.6 \%$ \\
\hline Left ventricular ejection fraction (\%) & $51.1 \pm 15.9$ \\
\hline$<30 \%$ & II.7\% \\
\hline $30-50 \%$ & $43.7 \%$ \\
\hline$>50 \%$ & $44.6 \%$ \\
\hline Hypertension & $47.4 \%$ \\
\hline Diabetes & $18.9 \%$ \\
\hline Dyslipidemia & $16.7 \%$ \\
\hline Atrial fibrillation & $41.8 \%$ \\
\hline Ventricular tachycardia & $20.1 \%$ \\
\hline History of heart failure admission & $23.4 \%$ \\
\hline
\end{tabular}

Abbreviations: NYHA, New York Heart Association. 
moderately decreased LVEF (30\%-50\%). However the prognosis of those with severely low LVEF $(<30 \%)$ was the lowest with frequent episodes of sudden cardiac death (Figure 3B). The 3-year incidence of sudden death was higher in patients with $\mathrm{LVEF}<30 \%$ than those with $\mathrm{LVEF} \geq 30 \%$ ( $15 \%$ vs $4 \%$, respectively, $\mathrm{p}<0.001)$. Primary prevention of sudden cardiac death with an implantable cardioverter defibrillator in those patients should be recommended when they meet the criteria in the authorized guidelines (Watanabe et al 2006). Recently, anemia has been emphasized as an important prognostic predictor in patients with CHF (Ezekowitz et al 2003). Our data also showed that anemia was significantly associated with all-cause mortality, cardiac-cause mortality, and sudden death in patients with diastolic CHF (Tada et al 2007), as well as in those with systolic CHF, as reported by other researchers (O’Meara et al 2006).

\section{Treatment of patients with CHF in Japan}

Treatments with angiotensin converting enzyme inhibitors (ACEI), angiotensin II receptor blockers (ARB), or $\beta$-blockers are recommended to improve prognosis and quality of life for patients with CHF (Hunt et al 2001). However it has previously been reported that such evidence-based treatments might not be sufficiently used in patients who should have had benefits of such medications (Masoudi et al 2003). The

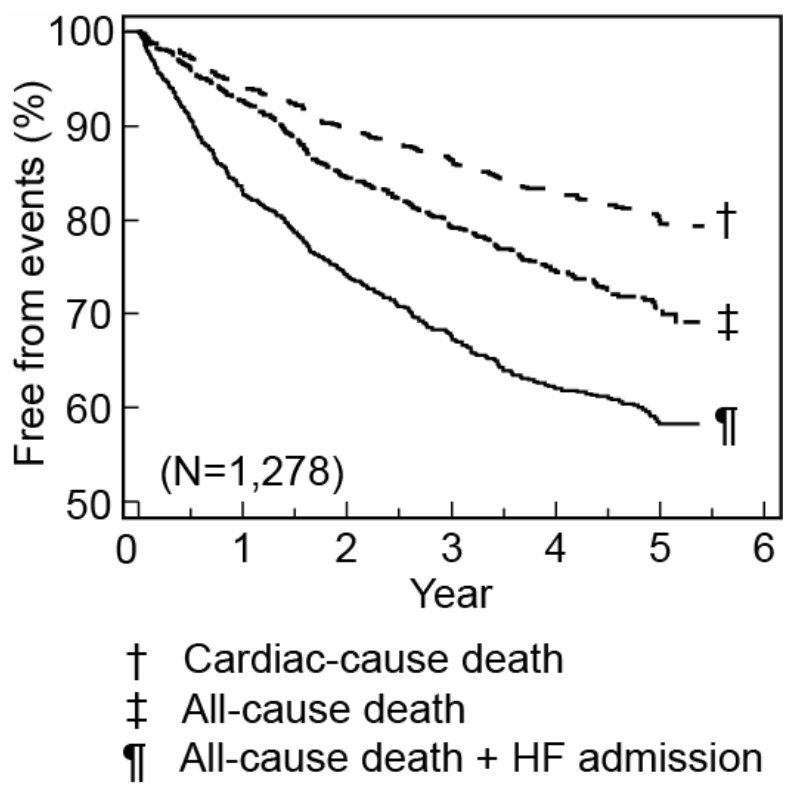

Figure 2 Prognosis of the Japanese patients with CHF in the CHART-I study. Copyright (C) 2004. Reproduced with permission from Shiba N, Watanabe J, Shinozaki T, et al. 2004. Analysis of chronic heart failure registry in the Tohoku district: third year follow-up. Circ J, 68:427-34.

Abbreviations: HF, heart failure.
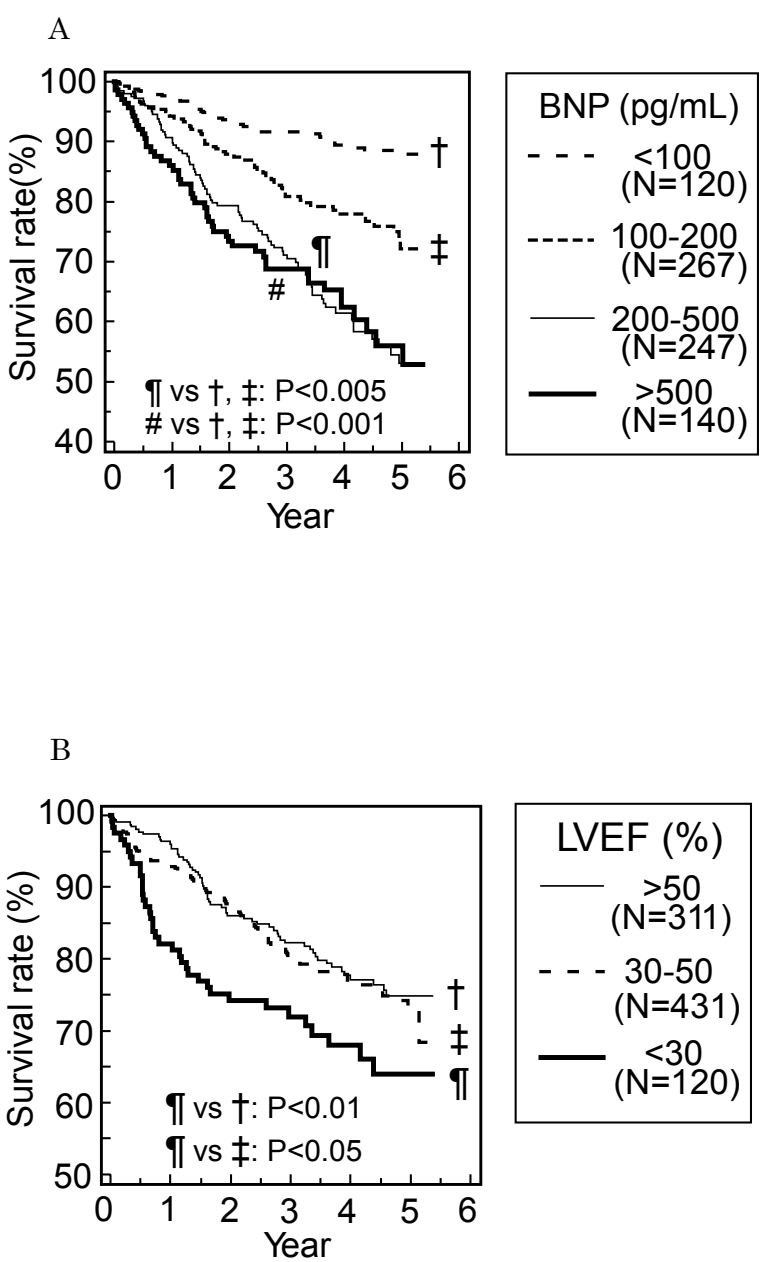

Figure 3 Kaplan-Meier curves of freedom from all-cause death stratified by (A) BNP and (B) LVEF in the CHART-I study.

Abbreviations: BNP, B-type natriuretic peptide; LVEF, left ventricular ejection fraction.

overall usage rate of $\mathrm{ACEI} / \mathrm{ARB}$ or $\beta$-blocker in patients enrolled in the CHART-1 study was $70 \%$ and $28 \%$, respectively (Figure 4). The penetration rate of these medications was relatively lower in female patients, elderly patients, and those with valvular heart disease or preserved LVEF, and this trend was more evident for the treatment with $\beta$-blocker than ACEI/ARB (Figure 4). These results suggest that future clinical trials are still necessary for such minorities who have not usually been enrolled in major randomized treatment trials for $\mathrm{CHF}$.

\section{Clinical outcomes of Japanese patients with $\mathrm{CHF}$}

Figure 5 showed survival curves of placebo groups in randomized treatment trials for CHF performed in Western countries, superimposed with the result obtained in our CHART-1 study with Japanese CHF patients. One-year allcause mortality of patients with mild-moderate CHF (NYHA 

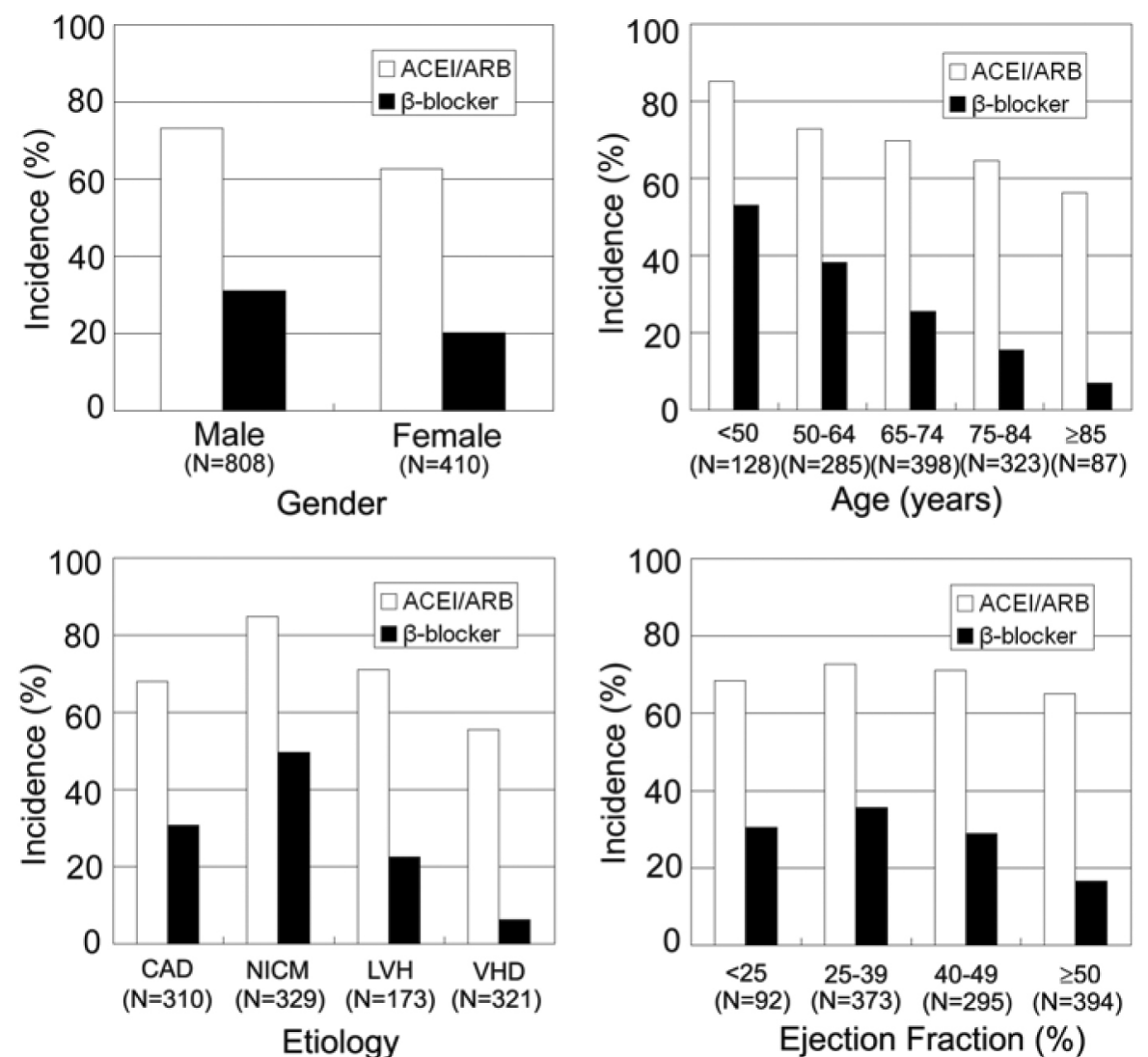

Figure 4 Prevalence of the use of renin-angiotensin inhibitors and $\beta$-blockers in the CHART-I study. Copyright @ 2007. Reproduced with permission from Shiba N, Takahashi J, Matsuki M. 2007. The CHART Study (Japanese). Naika, 99:4I0-I4.

Abbreviations: ACEI, angiotensin-converting-enzyme inhibitor; ARB, angiotensin II receptor blocker; CAD, coronary artery disease; NICM, non-ischemic cardiomyopathy; $\mathrm{LVH}$, left ventricular hypertrophy; VHD, valvular heart disease.

II-IV and LVEF $\leq 40 \%$ ) or moderate-severe CHF (NYHA III-IV and LVEF $\leq 35 \%$ ) was $13 \%$ and $21 \%$, respectively. Importantly, as is evident in Figure 5, the prognosis of Japanese patients with CHF was equally poor compared with Western CHF patients. Since the Japanese society is aging rapidly, a sharp increase in the number of $\mathrm{CHF}$ patients will be inevitable in the near future in Japan, as CHF is a disease of the elderly. Figure 6 shows the event rate in CHF patients based on age at the entry, demonstrating that elderly Japanese patients with $\mathrm{CHF}$ had an increased incidence of cardiac death and combination of cardiac death and admission due to congestive CHF. This is because elderly CHF patients have a higher rate of combined risk factors, such as anemia, chronic kidney disease, hypertension, and atrial fibrillation. Appropriate prevention strategies against the development and progression of CHF should be undertaken in Japan.

\section{Major CHF treatment trials in Japan}

There are 2 published randomized treatment trials for $\mathrm{CHF}$ patients in Japan (Table 3). The Multicenter Carvedilol
Heart Failure Dose Assessment (MUCHA) trial enrolled 174 patients with mild to moderate CHF to seek for the efficacy and optimum dose of carvedilol, with 3 treatment arms, including placebo, 5 and $20 \mathrm{mg}$ of the $\beta$-blocker in daily dose (Hori et al 2004). During the 24-48 weeks of the treatment period, carvedilol achieved dose-related improvement of the rate of death or cardiovascular hospitalization to $25 \%, 9 \%$, and $5 \%$ in the placebo, $5 \mathrm{mg}$, and $20 \mathrm{mg}$ group, respectively ( $p=0.002$ ). The Assessment of Response to Candesartan in Heart Failure in Japan (ARCH-J) study investigated the efficacy of candesartan ( $8 \mathrm{mg}$ once daily) in comparison with the placebo in 305 patients with symptomatic CHF (Matsumori et al 2003). During the 6-month follow-up period, fatal cardiovascular events occurred in 2 patients in each treatment group and the incidence of progression of CHF was $7 \%$ and $22 \%$ in the candesartan and the placebo group, respectively $(p=0.0004)$.

We have recently initiated a large outcome study with olmesartan in CHF patients, termed The Supplemental Benefit of Angiotensin II Receptor Blocker in Hypertensive 


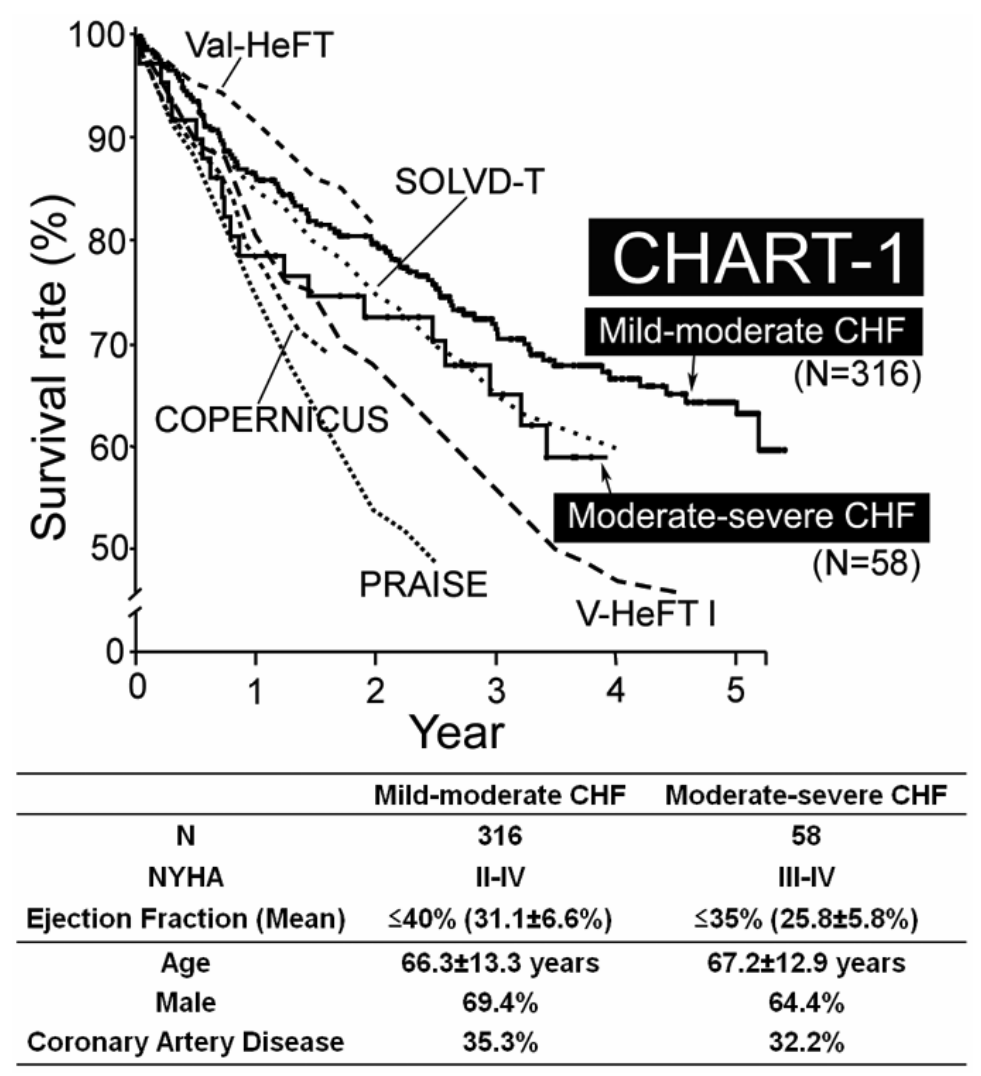

Figure 5 Comparison of the prognosis of patients with CHF between Western clinical trials and the CHART-I study. Copyright $\odot$ 2007. Reproduced with permission from Shiba N, Takahashi J, Matsuki M. 2007.The CHART Study (Japanese). Naika, 99:410-14.

Abbreviations: HYHA, New York Heart Association.

Patients with Stable Heart Failure Using Olmesartan (SUPPORT trial), which is currently the largest outcome study in Japan (Table 3). The purpose of our SUPPORT trial is to examine whether an ARB, olmesartan, in addition to conventional treatment, reduces the mortality and morbidity of hypertensive patients with stable CHF. The primary endpoint is a combined event of all-cause death, nonfatal acute myocardial infarction, nonfatal stroke, and hospital admission due to congestive heart failure. We also aim to evaluate the beneficial effect of olmesartan on the
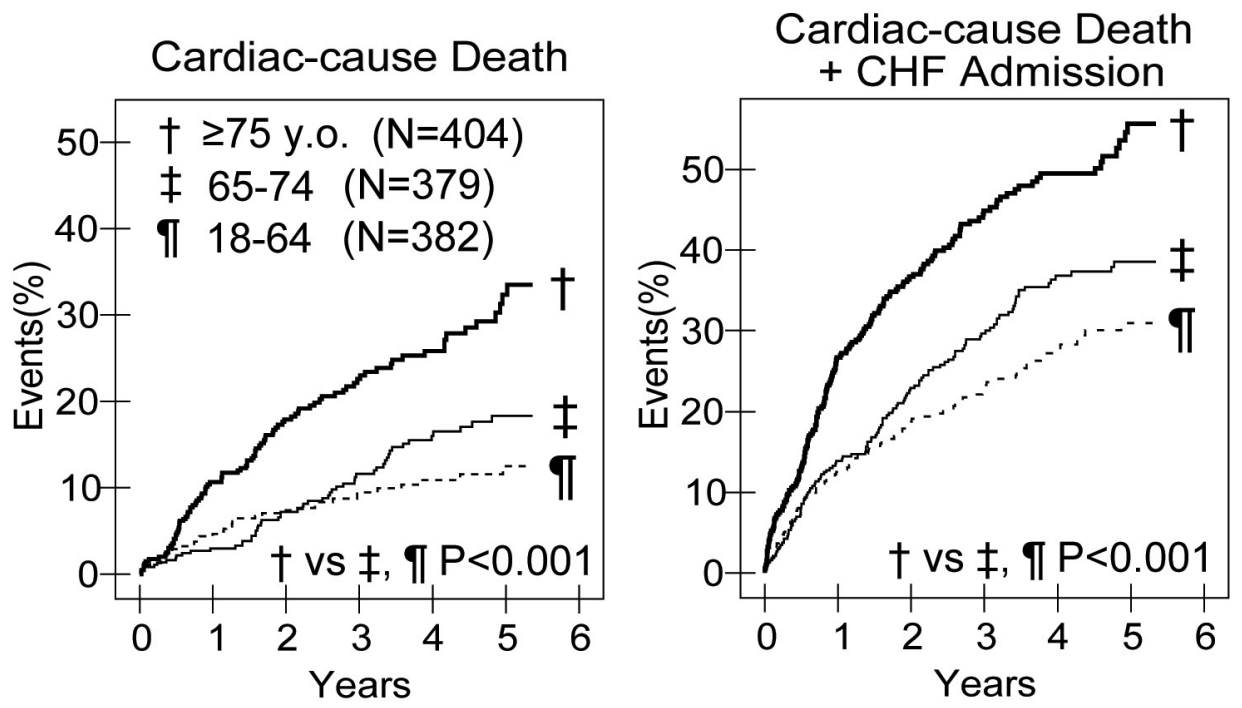

Figure 6 Prognosis of elderly patients with CHF in the CHART-I study. Copyright @ 2007 . Reproduced with permission from Shiba N, Takahashi J, Matsuki M. 2007. The CHART Study (Japanese). Naika, 99:4I0-I4.

Abbreviations: $\mathrm{CHF}$, congestive heart failure. 
progression of metabolic syndrome. The entry of patients was started in November 2007, and the results of the study will be obtained by the end of 2011 .

There are 5 other small outcome trials that are currently in progress in Japan (Table 3). The Assessment of BetaBlocker Treatment in Japanese Patients with Chronic Heart Failure (J-CHF) and the Japanese Diastolic Heart Failure Study (J-DHF) are investigating the effects of carvedilol in patients with systolic CHF and those with diastolic CHF, respectively. Another objective of J-CHF is to determine the optimum dose of carvedilol and to elucidate the differences in clinical characteristics between responders and nonresponders to the $\beta$-blocker. The Pitavastatin Heart Failure Study (PEARL study) is designed to evaluate the efficacy of pitavastatin for CHF with mild hypercholesterolemia. The Japanese Multicenter Evaluation of Long- versus short-acting Diuretics in Congestive Heart Failure (J-MELODIC) is designed to compare the effects of furosemide and azosemide in patients with CHF and to test the hypothesis that long-acting diuretics are superior to short-acting ones in those patients. The Japanese Heart Failure Outpatients Disease Management and Cardiac Evaluation Study (J-HOMECARE) was designed to evaluate the benefit of disease management program for prognosis, psychological status and quality of life of patients with CHF.

There are 2 large trials that have investigated the role of valsartan in Japanese patients with cardiovascular disease including CHF (Table 4). The Japanese Investigation of Kinetic Evaluation in Hypertensive Event and Remodeling Treatment (JIKEI-HEART) Study was designed to investigate whether concomitant treatment with valsartan in addition to conventional treatment improves the prognosis of Japanese patients with hypertension, ischemic heart disease, or congestive heart failure. The results of this study have recently been published (Mochizuki et al 2007). After a median follow-up period of 3.1 years, the incidences of stroke, transient ischemic attack, angina pectoris, and CHF were significantly lower in patients treated with valsartan compared with those with the conventional treatment. However, the benefit of the add-on valsartan treatment in a sub-population with $\mathrm{CHF}$, which accounted for $11 \%$ of the total population, has not been published yet. The Add-on Effects of Valsartan on MorbiMortality (KYOTO-HEART) study was designed to assess the add-on effect of valsartan on the conventional treatment in terms of the morbidity and mortality in Japanese hypertensive patients with high risks of cardiovascular diseases including CHF (Table 4).
Many of the randomized clinical trials performed in Japan utilize the prospective randomized open blinded endpoint (PROBE) design, as an alternative to the randomized double-blind placebo-controlled design (Tables 3 and 4). This is mainly because the PROBE study tends to be more cost effective and its open-labeled medication may minimize ethical considerations (Hansson et al 1992).

\section{Racial difference in morbidity and mortality due to cardiovascular diseases}

Several researchers have suggested that cardiovascular risk factors have different prognostic impact among different populations. The Seven Countries Study Research Group showed a substantial heterogeneity among populations in terms of the death rate due to coronary artery disease, even at a similar level of blood pressure (Van den Hoogen et al 2000). The proportion of deaths attributable to cardiovascular disease has also been reported to vary among different cohorts even at the similar serum cholesterol level. The longterm follow-up for 25 years for 12,763 men in 16 cohorts in Europe, the United States, and Japan showed that the risk factors of cardiovascular disease, such as insufficient physical activity and high serum cholesterol level, were not significantly associated with all-cause death in several countries including Japan (Menotti et al 2001). Since these differences cannot be explained by other baseline characteristics, smoking habits, or genetic difference, environmental and/or behavioral factors may play an important role in the development of cardiovascular diseases. The Ni-Hon-San Study, which compared cardiovascular disease rates and risk factors in Japanese men living in Japan, Hawaii, and California, showed that the mortality rate due to coronary artery disease was lowest in Japanese men living in Japan, whereas it was highest in those living in California (Benfante 1992). Racial differences may also influence the effect of medical treatment for CHF. Several studies demonstrated that genetic polymorphisms and/or a difference in $\beta 1$-receptor sensitivity, which are frequently observed in the Japanese population, might change the pharmacokinetics or the clinical effect of medical drugs, such as ACEI and $\beta$-blockers (Kubota et al 2000; Xie et al 2001; Ranade et al 2002).

\section{Future direction for the management of CHF in Japan}

CHF is a slowly progressive disease from stage A to stage D unless appropriately treated as described in the ACC/AHA guidelines (Hunt et al 2001). The strategy to manage CHF has been changing recently from treatment to prevention 


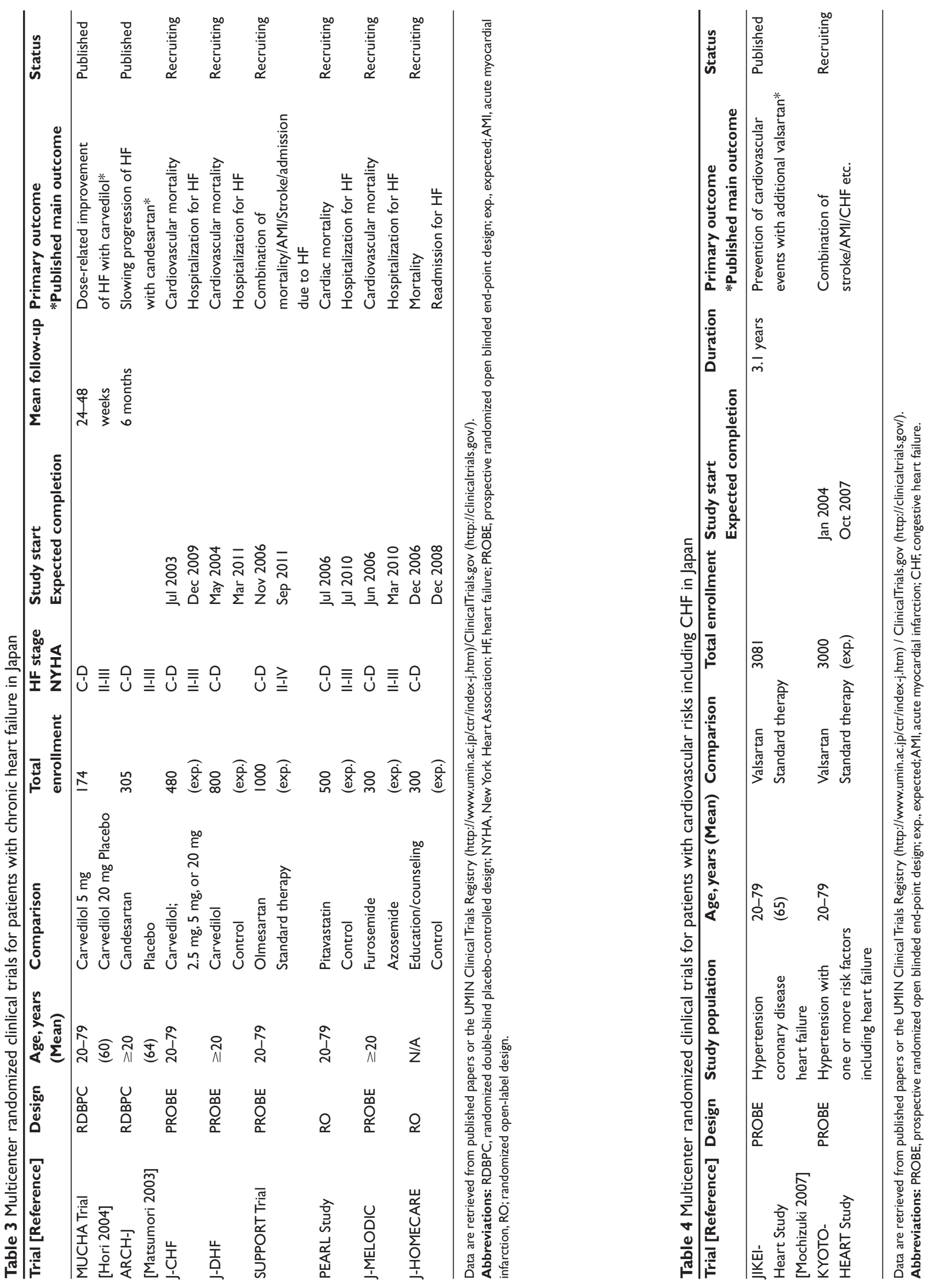


(Bansal et al 2006). Japanese CHF patients have several different profiles compared with Western CHF patients as follows; $(a)$ the prevalence of CHF of ischemic origin is lower, $(b)$ the percentage of elderly population is remarkably high, and $(c)$ the penetration rate of evidence-based medicine, such as ACEI/ARB or $\beta$-blockers, is not sufficiently high yet. The current situation of the management of $\mathrm{CHF}$ in Japan is probably caused by the fact that the number of randomized treatment trials for Japanese patients is not enough yet. Given the expected future increase in Japanese patients with $\mathrm{CHF}$, effective prevention strategy is necessary. Our on-going CHART-2 and SUPPORT studies will enable us to obtain effective strategies to improve the management of CHF in Japan.

\section{Conclusions}

The prevalence of CHF will rapidly increase in the next decades in many industrialized countries, including Japan. Large cohort studies with CHF patients are useful for risk stratification and determination of preventive measures for the disorder. Large-scale, randomized treatment trials also are needed, especially in Japan, in order to obtain further evidence to improve the management of patients with $\mathrm{CHF}$.

\section{Acknowledgments}

Active investigators of the CHART studies were described in the paper previously published (Shiba et al 2004) and at the website of the Tohoku Heart Failure Association (see http://tohoku.cardiovascular-medicine.jp). The CHART-1 study was supported by the research grants from the Ministry of Health, Labor and Welfare and Gonryo Medical Foundation. We are grateful for the assistance of research nurses who are working at the Tohoku Heart Failure Association; Mika Matsuki, Shizuka Osaki, Kiriko Yukishita, Yuuko Kidoguchi, Miho Hotta, Haruka Kohno, and Keiko Nishiura.

\section{References}

Bansal D, Chahoud G, Smith ES, et al. 2006. Prevention of heart failure. Curr Opin Cardiol, 21:510-16.

Benfante R. 1992. Studies of cardiovascular disease and cause-specific mortality trends in Japanese-American men living in Hawaii and risk factor comparisons with other Japanese populations in the Pacific region: a review. Hum Biol, 64:791-805.

Bettencourt P, Ferreira A, Dias P, et al. 2000. Predictors of prognosis in patients with stable mild to moderate heart failure. J Card Fail, 6:306-13.

Deedwania PC. 2003. The key to unraveling the mystery of mortality in heart failure: An integrated approach. Circulation, 107:1719-21.

Exercise and Physical Activity Reference for Health Promotion 2006. Office for Lifestyle-related Diseases Control, General Affairs Division, Health Service Bureau, Ministry of Health, Labour Welfare [online]. Accessed on June 10, 2007. URL: http://www.nih.go.jp/eiken/english/research/ program_exercise_epar2006.html.
Ezekowitz JA, McAlister FA, Armstrong PW, et al. 2003. Anemia is common in heart failure and is associated with poor outcomes: insights from a cohort of 12065 patients with new-onset heart failure. Circulation, 107:223-5.

Hansson L, Hedner T, Dahlof B. 1992. Prospective randomized open blinded end-point (PROBE) study. A novel design for intervention trials. Blood Press, 1:113-19.

Hori M, Sasayama S, Kitabatake A, et al. 2004. Low-dose carvedilol improves left ventricular function and reduces cardiovascular hospitalization in Japanese patients with chronic heart failure: the Multicenter Carvedilol Heart Failure Dose Assessment (MUCHA) trial. Am Heart $J$, 147:324-30.

Hunt SA, Baker DW, Chin MH, et al. 2001. ACC/AHA guidelines for the evaluation and management of chronic heart failure in the adult: Executive summary. J Am Coll Cardiol, 38:2101-13.

Ito M. 2004. Health Insurance Systems in Japan: a Neurosurgeon's View. Neurol Med Chir (Tokyo), 44:617-28.

Kubota T, Yamamura Y, Ohkawa N, et al. 2000. Frequencies of CYP2D6 mutant alleles in a normal Japanese population and metabolic activity of dextromethorphan O-demethylation in different CYP2D6 genotypes. Br J Clin Pharmacol, 50:31-4.

Life Expectancies at Specified Ages. Abridged Life Tables For Japan 2006, Ministry of Health, Labour and Welfare [online]. Accessed on June 10, 2007. URL: http://www.mhlw.go.jp/english/database/dbhw/lifetb06/1.html.

Masoudi FA, Havranek EP, Wolfe P, et al. 2003. Most hospitalized older persons do not meet the enrollment criteria for clinical trials in heart failure. Am Heart J, 146:250-7.

Matsumori A, ARCH-J study investigators. 2003. Efficacy and safety of oral candesartan cilexetil in patients with congestive heart failure. Eur $J$ Heart Fail, 5:669-77.

Menotti A, Blackburn H, Kromhout D, et al. 2001. Cardiovascular Risk Factors as Determinants of 25-year All-cause Mortality in the Seven Countries Study. Eur J Epidemiol, 17:337-46.

Mochizuki S, Dahlöf B, Shimizu M, et al. 2007. Valsartan in a Japanese population with hypertension and other cardiovascular disease (Jikei Heart Study): a randomised, open-label, blinded endpoint morbiditymortality study. Lancet, 369:1431-9.

O'Meara E, Clayton T, McEntegart MB, et al. 2006. Clinical correlates and consequences of anemia in a broad spectrum of patients with heart failure: results of the Candesartan in Heart Failure: Assessment of Reduction in Mortality and Morbidity (CHARM) Program. Circulation, 113:986-94.

Ranade K, Jorgenson E, Sheu WHH, et al. 2002. A polymorphism in the beta-1 adrenergic receptor is associated with resting heart rate. $\mathrm{Am} \mathrm{J}$ Hum Genet, 70:935-42.

Rector TS, Cohn JN. 1994. Prognosis in congestive heart failure. Annu Rev Med, 45:341-50.

Shiba N, Watanabe J, Shinozaki T, et al. 2004. Analysis of chronic heart failure registry in the Tohoku district: third year follow-up. Circ J, 68:427-34.

Shiba N, Watanabe J, Shinozaki T, et al. 2005. Poor prognosis of Japanese patients with chronic heart failure following myocardial infarction comparison with nonischemic cardiomyopathy. Circ J, 69:143-9.

Shiba N, Takahashi J, Matsuki M. 2007. The CHART Study (Japanese). Naika, 99:410-14.

Summary of Vital Statistics. 2005. Summary of Vital Statistics. Ministry of Health, Labour and Welfare [online]. Accessed on July 6, 2007. URL: http://www.mhlw.go.jp/english/database/db-hw/populate/index. html.

Tada T, Shiba N, Watanabe J, et al. 2007. Prognostic value of anemia in predicting sudden death of patients with diastolic heart failure. Int $J$ Cardiol, In Press.

The Status of Aging and Implementation of Measures for Aging Society in FY 2005. Annual Report on the Aging Society 2007, Cabinet Office [online]. Accessed on June 10, 2007. URL: http://www.cao. go.jp/index-e.html. 
Tsutsui H, Tsuchihashi-Makaya M, Kinugawa S, et al. 2006. Clinical characteristics and outcome of hospitalized patients with heart failure in Japan. -Rationale and design of Japanese Cardiac Registry of Heart Failure in Cardiology (JCARE-CARD)-. Circ J, 70:1617-23.

Tsutsui H, Tsuchihashi-Makaya M, Kinugawa S, et al. 2007. Characteristics and outcomes of patients with heart failure in general practices and hospitals. -Japanese Cardiac Registry of Heart Failure in General Practice (JCARE-GENERAL). Circ J, 71:449-54.

Van den Hoogen P, Feskens EJM, Nagelkerke NJD, et al. 2000. The Relation between Blood Pressure and Mortality due to Coronary Heart Disease among Men in Different Parts of The World. N Engl J Med, 342:1-8.
Watanabe J, Shiba N, Shinozaki T, et al. 2005. Prognostic value of plasma brain natriuretic peptide combined with left ventricular dimensions in predicting sudden death of patients with chronic heart failure. $J$ Card Fail, 11:50-5.

Watanabe J, Shinozaki T, Shiba N, et al. 2006. Accumulation of risk markers predicts the incidence of sudden death in patients with chronic heart failure. Eur J Heart Fail, 8:237-42.

Xie HG, Kim RB, Wood AJJ, et al. 2001. Molecular basis of ethnic differences in drug disposition and response. Annu Rev Pharmacol Toxicol, 41:815-50. 
